Mathematical Statistics and Limit Theorems 
Marc Hallin · David M. Mason

Dietmar Pfeifer - Josef G. Steinebach

Editors

\section{Mathematical Statistics and Limit Theorems}

Festschrift in Honour of Paul Deheuvels

第 Springer 


\section{Editors}

Marc Hallin

European Centre for Advanced Research in Economics and Statistics

Université Libre de Bruxelles

Bruxelles

Belgium

David M. Mason

Department of Applied Economics and Statistics

University of Delaware

Newark, DE

USA
Dietmar Pfeifer

School of Mathematics and Science

Carl von Ossietzky University

Oldenburg

Germany

Josef G. Steinebach

Mathematical Institute

University of Cologne

Köln

Germany

ISBN 978-3-319-12441-4

ISBN 978-3-319-12442-1 (eBook)

DOI $10.1007 / 978-3-319-12442-1$

Library of Congress Control Number: 2015933369

Springer Cham Heidelberg New York Dordrecht London

(C) Springer International Publishing Switzerland 2015

This work is subject to copyright. All rights are reserved by the Publisher, whether the whole or part of the material is concerned, specifically the rights of translation, reprinting, reuse of illustrations, recitation, broadcasting, reproduction on microfilms or in any other physical way, and transmission or information storage and retrieval, electronic adaptation, computer software, or by similar or dissimilar methodology now known or hereafter developed.

The use of general descriptive names, registered names, trademarks, service marks, etc. in this publication does not imply, even in the absence of a specific statement, that such names are exempt from the relevant protective laws and regulations and therefore free for general use.

The publisher, the authors and the editors are safe to assume that the advice and information in this book are believed to be true and accurate at the date of publication. Neither the publisher nor the authors or the editors give a warranty, express or implied, with respect to the material contained herein or for any errors or omissions that may have been made.

Printed on acid-free paper

Springer International Publishing AG Switzerland is part of Springer Science+Business Media (www.springer.com) 


\section{Foreword}

Paul Deheuvels is well known for his work in mathematical statistics and probability, especially in the area of limit theorems. Some of the topics on which he has made significant and lasting contributions are extreme and record value theory, renewal theory, copulas, strong approximations, Erdős-Rényi laws, empirical and quantile processes, nonparametric function estimation and Karhunen-Loève expansions. Through his consulting work Paul Deheuvels has also made substantial contributions to applied statistics.

Paul Deheuvels has had a major impact on statistics in France. Most importantly, in 1980 he founded the statistics research laboratory at the Université Pierre et Marie Curie in Paris (the LSTA, Laboratoire de Statistique Théorique et Appliquée), and served as its director until 2013. He has guided a large number of doctoral students. Many of them are now placed in prominent positions in academia and industry. Among other honours, Paul Deheuvels was elected Fellow of the Institute of Mathematical Statistics in 1985, and Membre de l'Académie des Sciences (France) in 2000 (Correspondent, 1996-2000). As the only statistician in the Académie, he has been a tireless supporter and promoter of statistics in France.

An overview of Paul Deheuvels' research and role as a force in statistics is given in Adrian Raftery's contribution to this Festschrift. A list of his publications is given at the end of the volume. 


\section{Preface}

During June 20-21, 2013 a committee of former students of Paul Deheuvels organized a conference on Mathematical Statistics and Limit Theorems in honour of his 65th birthday at the Jussieu Campus of the Université Pierre et Marie CurieParis VI. The committee consisted of

Salim Bouzebda (Université de Technologie de Compiègne, France)

Michel Broniatowski (Université Pierre et Marie Curie-Paris VI, France)

Sarah Ouadah (Université Pierre et Marie Curie-Paris VI, France)

Zhan Shi (Université Pierre et Marie Curie-Paris VI, France)

This volume is a collection of papers contributed by a selection of the invited speakers. Their topics are largely motivated by the research interests of Paul Deheuvels. The editorial board is grateful for the care that the contributors made in preparing their submissions, and acknowledges with thanks the efforts of the many referees who helped them in their editorial task.

Marc Hallin (ECARES, Université Libre de Bruxelles, Belgium)

David M. Mason (Department of Applied Economics and Statistics, University of Delaware, USA)

Dietmar Pfeifer (Institut für Mathematik, Carl von Ossietzky Universität Oldenburg, Germany)

Josef G. Steinebach (Mathematisches Institut, Universität zu Köln, Germany)

March 2015 


\section{Contents}

Paul Deheuvels: Mentor, Advocate for Statistics,

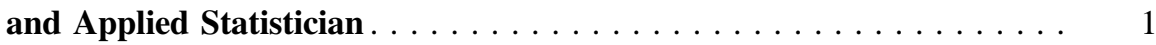

Adrian E. Raftery

Lacunary Series and Stable Distributions . . . . . . . . . . . . 7

István Berkes and Robert Tichy

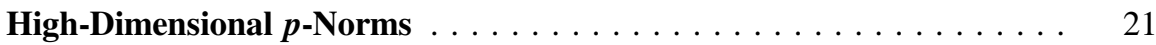

Gérard Biau and David M. Mason

Estimating and Detecting Jumps. Applications

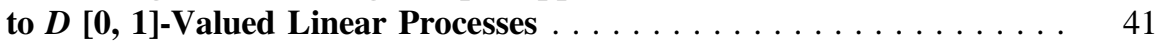

Denis Bosq

A Sharp Abelian Theorem for the Laplace Transform . . . . . . . . . . 67

Maëva Biret, Michel Broniatowski and Zansheng Cao

On Bahadur-Kiefer Type Processes for Sums and Renewals

in Dependent Cases . . . . . . . . . . . . . . . . . . . . . 93

Endre Csáki and Miklós Csörgő

Reduced-Bias Estimator of the Conditional Tail Expectation

of Heavy-Tailed Distributions. . . . . . . . . . . . . . . . . . . . . . 105

El Hadji Deme, Stéphane Girard and Armelle Guillou

On Sequential Empirical Distribution Functions for Random

Variables with Mean Zero . . . . . . . . . . . . . . . . . . . . . . . . . 125

Erich Haeusler and Stefan Horni 
On Quadratic Expansions of Log-Likelihoods and a General

Asymptotic Linearity Result.

Marc Hallin, Ramon van den Akker and Bas J.M. Werker

Asymptotic Normality of Binned Kernel Density Estimators

for Non-stationary Dependent Random Variables

Michel Harel, Jean-François Lenain and Joseph Ngatchou-Wandji

Approximation of a Random Process with Variable Smoothness . . . . 189

Enkelejd Hashorva, Mikhail Lifshits and Oleg Seleznjev

A Cramér-von Mises Test for Gaussian Processes.

Gennady Martynov

New $U$-empirical Tests of Symmetry Based on Extremal

Order Statistics, and Their Efficiencies

Ya. Yu. Nikitin and M. Ahsanullah

Optimal Rank-Based Tests for the Location Parameter

of a Rotationally Symmetric Distribution on the Hypersphere

Davy Paindaveine and Thomas Verdebout

Some Extensions of Singular Mixture Copulas . . . . . . . . . . . . . . . . 271

Dominic Lauterbach and Dietmar Pfeifer

Strong Laws of Large Numbers in an $\boldsymbol{F}^{\alpha}$-Scheme . . . . . . . . . . . . . . 287

Paul Doukhan, Oleg I. Klesov and Josef G. Steinebach

On Two Results of P. Deheuvels. . . . . . . . . . . . . . . . . . . . . . 305

Ju-Yi Yen and Marc Yor

Some Topics in Probability Theory . . . . . . . . . . . . . . . . . . . . 309

$\mathrm{Ju}-\mathrm{Yi}$ Yen and Marc Yor

Publication List of Paul Deheuvels—2014. 